\section{LONDON HOSPITAL.}

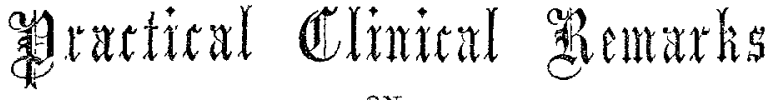 \\ ON}

\section{A C UTE PERIOSTITIS. \\ Delivered at the London Hospital,}

\section{BY T. B. CURLING, EsQ., F.R.S.,} SURGEON TO THE HOSPITAL.

GENTLEMEN,-There are few operations more frequently performed in the hospitals of London than those required for the removal of necrosed bone, and they are certainly more common now than they were in former years. The results show that these operations are essentially of a conservative character, that tedious sinuses, always discharging and liable to inflame, have been enabled to close, and that useful limbs have been saved by the removal of a constant source of irritation. These operations have become more frequent, then, because surgeons have become more strongly impressed with the advantage of extracting locked-up or impacted dead bone-have become less afraid of ill consequences from the necessary disturbance of parts, and have been emboldened to undertake tedious and troublesome operations, knowing that they can be rendered painless by chloroform. Our museums are rich in specimens of encased bone taken from amputated limbs; but such preparations are rarely added now, because it is seldom that a limb is removed for such a disease until the attempt to save it has been made by extracting the incarcerated bone. But so numerous are cases of necrosis in hospital practice that it is well to inquire whether we cannot, in many instances, prevent this serions result. The more common cause of necrosis in the long bones is acute periostitis, consequent upon injury, and the two following examples will illustrate the nature of this affection, and the mischief which results from the neglect of early and decisive treatment. The particulars of the first case are taken from the notes of the dresser, Mr. Michell.

CASE 1.-Acute periostitis of the femur, followed by a small necrosis. - Martha I__, a servant-girl, aged fourteen, was admitted into this hospital Oct. 27th, 1858. She stated that on the $23 \mathrm{rd}$ she was carrying a pitcher of water up some steps, when, owing to impaired vision consequent on nebule on the cornea after small-pox, she slipped and sprained her right knee. She slept but little that night, and next day was hot and feverish and unable to stand. The pain gradually became so acute that she could not bear to move her limb, and she continued to get worse until the period of her admission. At this time she had all the symptoms of acute sympathetic fever. The right lower extremity was much swollen, and she cried out with pain when the slightest pressure was made in the course of the femur, especially at its lower part. On the 28th I saw the patient for the first time. She had passed a sleepless night, and her symptoms were those above described. I could detect no fluctuation over the femur, but I at once determined to make an incision down to the bone at its lower and outer part. The integuments and vastus externus muscle were cut through, and the periosteum freely divided, when a small quantity of pus escaped, and it was ascertained by the finger that the membrane was detached from the bone. But little blood was lost. Pressure with a sponge was maintained for two hours, to prerent hremorrhage, and a poultice was after wards applied. She felt greatly relieved by the operation, and slept well the night afterwards. The febrile symptoms subsided quickly, and, supported with a generons diet, she soon regained her health. The wound, after discharging pus for a few days, gradually filled up, till at length only a small sinus was left. It was ascertained, on examination with a probe, that a small portion of the femur was denuded; but, as she was able to get about, she was discharged March 10th, 18.9, and directed, if the wround remained unclosed, to apply again for admission in three months' time.

She was readmitted in the May following. She was then in No. 1879 sood health, had grown stont, and the muscles of the thigh were well developed. There was a sinus furnishing a slight discharge, and a probe passed down to the femur seemed to enter an aperture and to grate against dead bone. On the 26 th I cut down to the bone, chiselled away a small portion, so as to enlarge the aperture, and extracted two small pieces of dead bone from the interior. 'l'here was no bleeding of any moment. The wound afterwards closed up readily. She was discharged July 18th, the sinus being nearly healed.

The notes of the next case are furnished by the dresser, $\mathrm{Mr}$. Welch.

CAsE 2.-Acute periositis of the tibia, followed by extensizi necrosis.-Mary Ann $\mathrm{C}$ - - , aged fifteen, a girl of strumous appearance, but who had generally enjoyed good health, was admitted into the hospitil on Feb. 14th, 1859. She stated that she had struck her left leg rather severely four days previously, since which it had become swollen, and excessively painful. Fomentations were ordered by the house-surgeon, and I saw her for the first time on the 17 th. She was suffering from high constitutional fever. Her pnlse was 200; indeed it could scarcely be counted; her tongue being dry and furred, and her cheeks flushed. She had no appetite whatever, and had not slept a minute for two nights. Her leg and ankle were greatly swollen and red in patches. There were great tension and extreme tenderness in the direction of the tibia. I at once made a long and free incision over the bone, when a large quantity of pus immediately escaped with considerable force. The finger passed into the wound, came in contact with the tibia exten. sively denuded. She was greatly relieved by the incision, and the fever subsided rapidly afterwards. Abscesses, attended with a good deal of suffering and constitntional disturbance, formed subsequently over both malleoli. They were opened, and found to be connected with the bone. Her health became so much impaired, and her strength so reduced, and there was so much swelling above the ankle, with indications of the joint being affected, that amputation seemed to be called for. I had her placed, however, under the influence of chloroform, and then, on careful examination, finding the ligaments tolerably firm, and being unable to detect any crepitus, I determined to watch the case a little longer. Some improvement was shortly manifested, thongh her recovery was retarded by the formation of another abscess over the internal malleolus, which was also opened. The improvement afterwards continued, and by the end of March, under tonics and a generous diet, the patient's strength was greatly restored. The wounds closed up a good deal, leaving numerous sinuses leading down to dead bone, with healthy granulations. The head of the tibia appeared to have retained its healthy state, but the rest of the bone gradually became greatly enlarged, the thickening extending to the inner malleolus.

June 30th. - I laid open some of the sinuses, chiselled away a quantity of soft new bone, and removed several pieces of dead bone. The necrosis was irregular, consisting, in some places, of a thin scale from the surface; in others, of nearly the whole thickness of the tibia encased in new bone, and in some places of small portions of the interior enclosed in the original bone, thickened, and soft in texture. One of the latter was extracted quite from the lower end of the tibia, and this communicated with the sinus over the internal malleolus. Since this opera. tion the patient has gone on extremely well. Her general health has improved; the sinus over the malleolus has closed ap; the enlargement of the tibia has subsided, and the large wound has steadily healed up.

When we consider that the whole, or greater part, of a bone may be destroyed by acute periostitis--that the inflammation may extend to the adjoining articulations, imperilling the safe's of a limb, and that patients sometimes sink under the constitutional fever attending it, I need not nurge the importance of an early diagnosis of the disease, in order that right and prompt measurcs may be taken for its removal. The complaint for which acute periostitis is most liable to be mistaken is acute rheumatism; and it is a mistake which, I fear, is not unfrequently made in practice. Indeed, some care and nice observation are required to make the diagnosis. In rheumatism, as in periostitis, there is high inflammatory ferer, with swelling of the limb, and great pain, increased by pressure, so that the patient is nearly helpless, and he shrinks from the touch of the surgeon, in dread of the torture which an examination may cause him. In periostitis, say of the femur or tibia, the swelling is diffused. It is not limited to the larger joints-to the ankle or to the knee, but occupies a wider range, and is cdematous in character. But the chief diagnostic mark is the seat of pain. In periostitis little or no pain is caused by pressure, unless it be made over, or in the course of, the affected bone. You may, 
in the early stage, move the limb at the linee or the ankle, and press the ligaments and tendons without producing pain, but the slightest pressure on the bone excites intense suifering. you press over the tibia or the muscles of the thigh around the femur in rheumatism, you rarely cause much pain; but in acute periostitis such pressure cannot be tolerated for a moment. The conclusion in favour of periostitis will be much strength. ened if it be found, as in the cases just related, that the attack of inflammation succeeded an injury.

The treatment commonly recommended in acute periostitis is local depletion with calomel and opium. Just at the onset of an attack, in a superticial bone like the tibia, this treatment may be of service, but in periostitis of a deep-seated bone, or if the inflammation do not speedily subside, such measures are not to be relied on. After matter has formed beneath the membrane, they are worse than useless. They weaken the patient without exerting any infuence on the disease. There is then no way of averting serious mischief but by a free in cision of the inflamed periosteum. Some of you evinced surprise when, in the case of $\mathrm{L}$ _ on my first visit to the patient, though no fluctuation was perceptible, I determined at once to cut boldly through the thick vastus externus muscle down on the femur. There was, indeed, no other mode of preserving the bone. I was satisfied, after inquiring into the history of the case, and a careful examination of the limb, that the perios teum of the femur was acutely inflamed, and the incision revealed the presence of a small quantity of pus confined beneath it. The membrane was partially detached, and in a few hours extensive mischief would have ensued. This was arrested by the incision, and the almost immediate cessation of suffering, the rapid subsidence of the inflammatory fever, and the speedy restoration to health, were the satisfactory results of this decisive treatment. What would have been the condition of the patient had the operation been delayed, may be learnt from the second case. I did not see C-until after a large suppuration had taken place. The periosteum was extensively detached, and so distended by the pus effused beneath it, that when an opening was made, the matter gushed out with force over my clothes. In the first case, notwithstanding the early relief by an incision, slight death of bone ensued; but in the second case the necrosis was very extensive, and the inflammation having affected the cancellous tissue in the lower end of the tibia, the ankle-joint became at one time in jeopardy. Additional incisions were required, and under this treatment the urgent symptoms at length subsided, the inflammation in the periosteum and bone, and the necrosis, ceased to extend, the work of restoration was set up, and the case had a favour. able issue.

\section{ANEURISH OF THE POPLTTEAL ARTERY SUCCESSFULLY TREATED BY FLEXION AND COMPRESSION.}

By OLIVER PEMDERTON, EsQ., M.R.C.S.,

SUBGEOX TO THE GEYERAL HOSPITAL, AND LECTURER ON SUTGICAI PATHOLOGT AT SYDENHAIT COLLAGE, BIRMITGHAM.

THE mode of treatment adopted for the cure of popliteal aneurism in the following case exhibits a new feature in the treatment of this deeply interesting and important surgical malady, and from the success which has attended its applica. tion, I am led to think that it may not be found unworthy of the attentive consileration of the profession.

J. $\mathrm{K}-$, aged twenty-two, a native of Bombay, possessing all the characteristics of his country, was admitted nnder my care into the Birmingham General Hospital on May 10th, 1859 , with a tumone in the left ham.

On examination, the swelling was found to be an aneurism of the popliteal artery. It was very large, measuring five inches in its willest diameter. It occupied the entire popliteal space, and projected very much laterally. The pulsation was marked, and distinctly visible at a considerable distance. The hand, laid on the tumour, experienced an appreciable thrill, whilst the stethoscope detected a loud systolic and a somewhat feebler diastolic bruit-not only over the aneurism, but also along the course of the femoral artery. Pressure on the femoral, at the unbic arch, commanded the flow of blond into the tumour, which at once collapsed and disappeared, and then rapidly refilled on the compression being removed. There was no other arterial disease to be discovered, and the heart's sounds were natural.
All the history he could give of himself was, that he had been in this country for the past twelve years, getting his living by hawking small articles from place to place. He had walked a great deal of late, and had noticed the swelling only four months before admission. He thought it must have come of its own accord, as he did not recollect having fallen or received injury on the part. The man was of small stature, lithsome in frame, and possessed the well-defined muscles, free from fat, peculiar to his countrymen. He walked with a limp, and complained of pain on forced flexion of the limb, but hand ling the swelling did not appear to trouble him. He whined and moaned, and from his general irritability promised anything but a calm patient for operative interference.

Having discussed the features of the case in consultation with my colleagues, $T$ determined on endeavouring to cure the disease by the combination of compression and flexion. Accordingly, in order to accustom him to the influence of the instrament, the mode of treatment by compression was commenced on the 13th of May, the lower yad of Weiss's compressor being lightly fixed on the artery, just as it enters its aponeurotic canal. In a few hours, however, the pressure was removed, as he became restless and irritable.

I now permitted bim to smoke as often as he liked, in the hope that his disposition might become somewhat more tractable under the soothing influence of the cigarette. In this anticipation I was not disappointed, the lapse of a day or two sufficing to render him cont $\epsilon$ nted and obedient.

On the 16th of May, (having made arrangements by means of relays of dressers that he should never be left for eight-andforty hours, ) at twelve o'clock at noon, I fixed Weiss's com. pressor, and applied pressure by means of the lower pad to the middle third of the artery; at the same time, turning a bandage around the ankle-joint, I bent the leg as far as it would admit withont occasioning pain, and fixed it firmly in this posi. tion by carrying the bandage around the pelvis.

The effect of this combination of flexion and compression was, that the pulsation in the aneurism was reduced to a mere wave, varying from time to time, as the compressing pad was adjusted by the attendant.

Two hours after the commencement of this treatment he became very restless, and complained of a burning pain in the aneurism and down the shaft of the tibia; and, despite the permission to smoke, it was with much difficulty he could be kept quiet. Forty drops of the sedative solution of opinm were given at five o'clock with good effect.

Towards midnight, between eleven and twelve hours after the commencement of the treatment, he became much quieter, and slept frequently. The pressure exercised by the tourniquet was comparatively slight, the aneurismal tumour having become hard and perfectly free from pulsation. Not the least movement of the leg from the state of flexion had, however, been permitted. There was considerable swelling of the knee, leg, and foot, but he did not complain of much pain. It was remarkable to observe the vehement pulsation of the superficial arteries after the arrest of the circulation through the tumour, esperially about the neighbourhood of the internal articular vessels. During the night, the pressure on the artery was removed, from time to time, from the lower to the upper pad; but the amount exercised was merely nominal, as it was guite evilent that no blood had passed through the aneurismal tumour after the first twelve hours. A drachm of the sedative solution of opinm was administered towards morning, to the great comfort of the patient, who was calm and placid, chatting with his watchers and smoking in the interval of his slumbers.

Throughout the day of the $17 \mathrm{th}$, the same regulations were carried out, without the least deviation, and no annoyance was complained of from the position of the knce.

On the 18th, at twelve ocluck, forty-eight hours from the commencement of the treatment, I removed the compressor, and discontinued the supervision of the dressers. I made no relaxation whatever in the flexion of the knee. There was considerable swelling of the extremity, but no diminution in warmth. I had it carefully wrapped in cotton wool, and warmly covered up with flannel. There was good pulsation in the malleolar vessels, and not the least pulsation to be detected in the aneurism, or bruit along the course of the femoral artery. The articular vessels pulsated strongly.

On the 19th, the flexed position was slightly relaxed, and the eutire limb rolled with flannel.

24th.-Further liberty was allowed. The tumour has lost its lateral bulk, and begins to contract. The swelling of the leg is subsiding.

28th. - Contraction going on in the aneurism. The bandage between the ankle and pelvis still more relaxed, so as to permit 